\title{
Energy Monitoring and Management Mechanism for Wireless Sensor Networks
}

\author{
Andreas Papadakis ${ }^{1, a}$ and Theodore Zahariadis $^{2}$ \\ ${ }^{1}$ Department of Electrical \& Electronic Eng. Educators, School of Pedagogical and Technological Education, Athens, Greece \\ ${ }^{2}$ Department of Electric Engineering, Technological Education Institute of Sterea Ellada, Chalkida, Greece
}

\begin{abstract}
In this work we discuss a mechanism for the monitoring and management of energy consumption in Wireless Sensor Networks. We consider that the Wireless Sensor Network consists of nodes that operate individually and collaborate with each other. After briefly discussing the typical network topologies and associating with the expected communications needs, we describe a conceptual framework for monitoring and managing the energy consumption on per process basis.
\end{abstract}

\section{Introduction}

Sensor Networks consist of spatially distributed nodes, typically equipped with sensors which monitor physical conditions. The measurements and data retrieved form the WSN are made available to a server - based application through the Gateway.

Operations in a WSN are related to the actual retrieving of measurements and their processing. The processing of the measurements may also include adaptation or aggregation. At some point the data / information is communicated either from one node to another or to the centralized applications. Collaborations scenarios do exist and in principle facilitate the operations of the WSN.

When compared to traditional ad hoc networks, the most noticeable point about sensor networks is that they are limited in power, computational capacities, and memory. Indeed, assuming the typical operation of the network, i.e. without unexpected events, the lifetime of the network is depending on the energy availability in the nodes. While nodes typically consume limited energy, this consumption level can be customized, in relation to the measuring (sensing) operation, the internal functions that take place and the management of the communications activities.

Especially for the communications part, and assuming that the sensors can interchange idle and active modes, difference in the energy consumption can be significant. This means that there is margin of improving the performance of the WSN in terms of energy consumption.

Indeed such an improvement or even optimization of the energy consumption in wireless sensor networks is becoming a key performance objective. The objective is to maximize sensor network lifetime, without harming

\footnotetext{
${ }^{\text {a }}$ Corresponding author: author@e-mail.org
}

the performance of the network. The performance of the deployment does depend on the monitoring regions, the battery capacity and the energy consumption rate for each sensor.

The main task of a sensor node in a sensor network is to monitor events, i.e. collect data, perform quick local data aggregation, and then transmit the data. Power consumption can hence be divided into three domains: sensing, aggregation, and communication. In this paper we mostly concentrate on minimizing energy for communications [1].

These form a problem difficult to handle. In order to form a methodological approach of such a problem we need a framework for the monitoring and the management of power consumption. This is the objective of our work. Without restricting the generality of the WSN operations, we attempt to categorize and formulate a framework for understating the energy consumption attributes.

The structure of the paper is as following: In Section II, we discuss the typical topologies of the system along with the more important characteristics. In Section III we present the framework for associating the network topology with the power consumption. Section IV includes a short discussion on monitoring and enforcement mechanisms. The paper closes with the brief discussion in Section V.

\section{Topologies}

A sensor network is typically composed of a configurable number of sensor nodes deployed in the area that has to be monitored. More frequently than not, the positions of the sensor nodes are predetermined. The selection of the location of the nodes depends on the way the interested area is covered as well as other characteristics including 
access, the needs for data relaying, the efficiency and redundancy of covering the monitored area. On the other hand this is not strictly mandatory as we can have random deployment in inaccessible terrains or hazardous environments. The density and the number of nodes must ensure that the event is detected and forwarded to the gateway with a suitable probability of success while maintaining a low probability of false alarm.

In this Section we discuss the typical topologies that can be selected for the deployment of the sensors. The selected topology is a key aspect for the communications scenarios and affects the energy consumption. We consider that the network consists of the sensor nodes and the Gateway that connects the WSN with an external network (e.g., a local area network, an intranet or the Internet) that accommodates the centralized application (which monitors and manages the wireless sensor network). While multiple gateways can collaborate, for our consideration we include only one Gateway.

The typical topologies used in WSNs include (a) the point to point topology, (b) the star topology, (c) the mesh topology and (d) the hybrid topology. Hybrid configurations can also take place, combining characteristics from different topologies [2].

The point-to-point topology consists of dedicated wireless links between two sensor nodes. Its main characteristic is the single data communication channel per each couple of nodes. The fact that we have a single channel allows for a secure path but it also consists a single point of failure, i.e. if this path is broken no communication can take place. Such a topology is considered monolithic as it does not allow for selection of routes. The energy consumption can be predetermined and depends on the frequency of communications.

In the star communication topology, the nodes only send information to the central node (which is typically the gateway) through one hop. This is a quite simple and straightforward topology which does not include the possibility of communications among nodes. The star topology is characterized by flexibility and scalability. The central node (the gateway) composes a single point of failure. In case of failure, the nodes are disconnected from the external network.

The sensors transmit when information for the gateway is available. The nodes remain at sleep mode until the time they need to communicate the event to the gateway. The sensors also send in a periodic or aperiodic fashion messages to the gateway indicating that they are still functioning properly.

Nodes participate in bidirectional communication only with the gateway. In this case the nodes should also be able to receive information from the gateway. Thus a scheme is needed so that the nodes wake up at predefined instances in order to listen to messages coming from the gateway. These messages are needed so that the gateway keeps a global view of the state of the network. In terms of energy, the consumption scenarios are also predetermined (as the star topology is an extension of the point-to-point topology) and the actual consumption depends on the frequency of the need for communications.

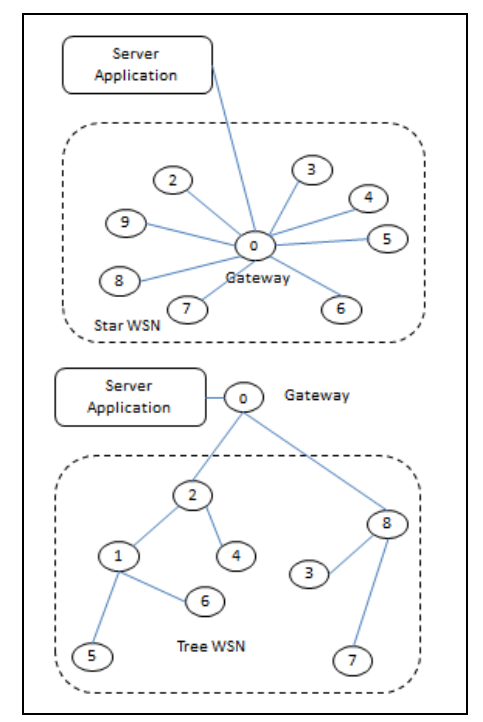

Figure 1. Star and tree topologies.

The tree topology is more complex than the star one. The deployed sensors create a logical tree when we talk about tree topology. We have two types of nodes; one is parent (data relay) node and other is leaf node. Data can be passed from a leaf node to its parent nodes and vice versa. The tree topology includes communication among nodes and the gateway and between nodes. In this case some of the nodes cannot reach the gateway through one hop communication but instead rely on multi-hop communication. Every node is assigned a neighbour as its parent and forwards to that the data intended for the gateway. The gateway is typically the root of the tree. The root (the gateway) forms a single point of failure but the possibility of multiple paths to connect one node with another allows for some robustness.

However, if a parent node fails, then its entire sub-tree is cut off from the base station unless the child nodes can find alternative routes to the gateway through other neighbours. Deep tree topologies incur long delays for sending data from leaf to root node as data will go through all the nodes of that group [2].

The dynamic situation regarding the status of the nodes leads to shallow trees, i.e. with a limited number of levels. The possibility to make selections regarding the route to follow (and the subsequent power needed) allows for flexible power management. The nodes closer to the base station consume more power in forwarding packets from all the nodes in their sub-parts, whereas the leaf nodes in the tree do not have to perform any forwarding at all and consume the least power.

The mesh topology is a multi-hoping system in which each node can communicate directly with each other node. In this case we do not have a single point of failure and it can be considered a reliable and scalable communication network structure. The redundancy in routing possibilities makes the network more robust.

Alternate paths can be selected depending on the dynamic status of the nodes and the communications path. However for efficient operation of a mesh network, the nodes should listen most of the time (if not continuously) and this leads to increased level of energy 
consumption. The lack of a more formal topology also creates increased latency.

\section{Communications Consumption}

\section{and Energy}

In this Section we briefly discuss the typical communications needs that are expected in a Wireless Sensor Network and the way they can be associated with the energy needs for the nodes of such a network.

\subsection{Communications needs}

The communications needs in a typical wireless sensor network consist of different traffic categories. We consider the following types of traffic (a) notification / alerting, (b) topology formulation control messages and signalling, (c) retrieval and sharing of measurements and (d) control messages coming from the centralized application towards the nodes.

Each of these types of traffic has its own characteristics. The most important characteristics include the frequency of message exchange and the average length of each message. These two, their product, gives the expectation of the average volume of traffic.

In order to coarsely approach the consumption for communications we consider the concept of the Duty Cycle. Duty Cycle is considered as the percentage of the active period (for potentially transmitting / receiving) towards the overall cycle.

The length of the message per case depends on its type. For notification, alerting, control and signalling, messages are brief. Their length can be realistically expected at the area of dozens or a hundred of Bytes (depending also on the overhead of the communications protocol).

On the other hand, for the retrieval and sharing of the measurements, the message length can be increased. This of course depends on the type of the measurement. For example scalar measurements can be expected to create brief messages, while visual monitoring is expected to create more heavy traffic. In such a case each message may include a frame, typically compressed if the node supports a compression algorithm, or uncompressed.

The duration of the transmission depends on the bandwidth achieved in the communications among nodes and among the nodes and the gateway. The duration of each individual transmission is equal to the division of the message length to the bandwidth. This figure is multiplied by the number of links that the message is expected to traverse until it reaches its final destination.

Table 1. Communications characteristics.

\begin{tabular}{|l|c|c|c|}
\hline \multirow{4}{*}{ Char/stic } & \multicolumn{3}{|c|}{ Types of Messages } \\
\cline { 2 - 4 } & $\begin{array}{c}\text { Alert, } \\
\text { notification, } \\
\text { control } \\
\text { command }\end{array}$ & $\begin{array}{c}\text { Routing } \\
\text { message }\end{array}$ & $\begin{array}{c}\text { Measurements } \\
\text { (especially in } \\
\text { the case of } \\
\text { visual signal) }\end{array}$ \\
\hline Length & $\begin{array}{c}\text { Brief }(\sim 10 \mathrm{~s} \text { of } \\
\text { Bytes })\end{array}$ & $\begin{array}{c}\text { Brief } \\
(\sim 10 \mathrm{~s} \text { of } \\
\text { Bytes })\end{array}$ & $\begin{array}{c}\text { Long }(\sim 10 \mathrm{~s} \text { of } \\
\text { KBytes })\end{array}$ \\
\hline
\end{tabular}

\begin{tabular}{|l|c|c|c|}
\hline Frequency & $\begin{array}{c}\text { Depending on } \\
\text { application }\end{array}$ & $\begin{array}{c}\text { Periodic } \\
\text { or upon } \\
\text { request }\end{array}$ & $\begin{array}{c}\text { Depending on } \\
\text { application }\end{array}$ \\
\hline Priority & High & Medium & Medium \\
\hline
\end{tabular}

We consider, as an example, the length of a short message to be equal to 100 Bytes (including the headers and the payload) and that of a long message to be around $77 \mathrm{~KB}$, assuming the exchange of a VGA frame with 2 bits for colour. We also assume a realistic bandwidth value of $50 \mathrm{kbps}$, equal to approximately $6.25 \mathrm{KBps}$ (Kilobytes per second).

In this case and dividing the size with the bandwidth we take approximate values for transmission duration: (a) $16 \mathrm{msec}$ for transmission of short messages (per hop) and (b) $12.3 \mathrm{sec}$ for transmission of long message (per hop).

\subsection{Connection of communications with energy consumption}

The general framework for associating the communications needs with the power consumption is to consider the duty cycle. Such duty cycle - based approach can allow for energy effective communications activities. The duty cycle approach requires the node to be deactivated between two consecutive communications activities.

The concept of the duty cycle can allow for runtime adaptation to meet lifetime requirements or energy availability. While this adaptation can be applied to the whole portfolio of sensor activities e.g. by adjusting sampling rates, we focus on a flexible mechanism monitoring (and potentially) managing the energy consumption.

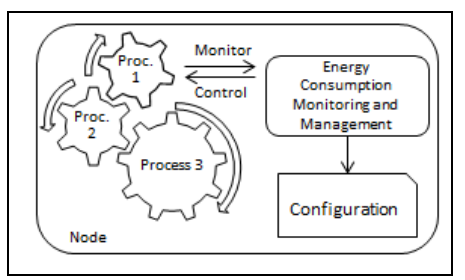

Figure 2. Energy consumption monitoring and management

We formulate a component - based decomposition that is common for architectures managing scarce shared resources. This includes (a) a mechanism to monitor the system and component energy usage, and (b) a management module for enforcing policy / configuration directives. These compose the Energy Consumption Monitoring and Management (ECMM) module as depicted in figure 2.

The Energy Consumption Monitoring and Management framework allows to view and potentially influence the energy budget of a node. Per each node system the energy consumption is calculated based upon the duty cycle of each component.

The energy monitoring and the configuration parameters allow the ECMM module to make estimations and decisions. For example, the average consumption rate can be calculated to estimate the remaining lifetime. 
This affects the operation at the level of each individual (and autonomous) sensor.

At the level of the WSN, the administrator can monitor and manage resource usage by sharing system energy levels amongst nodes. The co-ordination entity can be considered as intrinsic to the WSN (e.g. residing on the Gateway or distributed per node) or belonging to the application-specific implementation.

\section{Discussion - Conclusions}

Sensor networks are becoming more widely adopted for commercial and scientific use and, in settings where battery replacement or recharging is difficult, it is important that the networks have long and predictable lifetimes. Energy management does play a role in meeting user requirements. In principle system developers seek to achieve balance between network lifetime and performance.

In this work, which is in progress, we have set the framework for approaching and associating the energy consumption with the wireless sensor communication activities.

We have considered the main topologies and their high-level implications regarding the need for communications for different types of traffic. The communications needs identified (including notification, alerting, control, signalling and measurement retrieval) constitute the types of traffic in the majority of the WSN. One key issue coming from our analysis is related to the fact that these traffic types can be handled in an individualized manner.

Employing the concept of the duty cycle we have formulated a module that monitors and potentially manages the percentage of activity period of the involved processes, adjusting the relevant duty cycle. In this sense the node can decide either on its own or on a collaborative (among nodes) or orchestrated (by the server application) basis, and considering the configuration possibilities, the energy policy that will be followed.

\section{References}

1. Berman, P., Calinescu, G., Shah, C., \& Zelikovsky, A., Efficient energy management in sensor networks. Ad Hoc and Sensor Networks, Wireless Networks and Mobile Computing, 2, 71-90 (2005).

2. S. Sharma, D. Kumar, K. Kishore, Wireless Sensor Networks-A Review on Topologies and Node Architecture, International Journal of Computer Sciences and Engineering. Vol. 1(2) (2012).

3. Jiang, X., Taneja, J., Ortiz, J., Tavakoli, A., Dutta, P., Jeong, J., Shenker, S., An architecture for energy management in wireless sensor networks, ACM SIGBED Review, 4(3) (2007)

4. W. Dargie, Dynamic power management in wireless sensor networks: State-of-the-art, Sensors Journal, IEEE, 12(5), 1518-1528 (2012) 